\title{
Vorticity Statistics In The Two-Dimensional Enstrophy Cascade
}

\author{
Jérôme Paret, Marie-Caroline Jullien and Patrick Tabeling \\ Laboratoire de Physique Statistique, Ecole Normale Supérieure, \\ 24 rue Lhomond, 75231 Paris (France)
}

(June 6, 2018)

\begin{abstract}
We report the first extensive experimental observation of the two-dimensional enstrophy cascade, along with the determination of the high order vorticity statistics. The energy spectra we obtain are remarkably close to the Kraichnan Batchelor expectation. The distributions of the vorticity increments, in the inertial range, deviate only little from gaussianity and the corresponding structure functions exponents are indistinguishable from zero. It is thus shown that there is no sizeable small scale intermittency in the enstrophy cascade, in agreement with recent theoretical analyses.
\end{abstract}

The enstrophy cascade is one of the most important processes in two-dimensional turbulence, and its investigation, at fundamental level, provides cornerstones for the analysis of atmosphere dynamics. The existence of this cascade was first conjectured by Kraichnan [1], and later by Batchelor [2]. Both of them proposed that in two-dimensional turbulence, enstrophy injected at a prescribed scale is dissipated at smaller scales, undergoing a cascading process at constant enstrophy transfer rate $\eta$; this led to predicting a $k^{-3}$ spectrum for the energy, in a range of scales extending from the injection to the dissipative scale. Later, logarithmic corrections have been incorporated in the analysis to ensure constancy of the enstrophy transfer rate [3]. The advent of large computers revealed surprising deviations from the classical expectation, especially in decaying systems 99, 10,7]. It was soon realized that in two-dimensional systems, long live coherent structures inhibit the cascade locally and therefore the self similarity of the process, assumed to fully apply in the classical approach, is broken. Expressions like "laminar drops in a turbulent background" were coined to illustrate the role of coherent structures in the problem [9]. Along with the observations of unexpected exponents, models, emphasizing on the role of particular vortical structures [12,11, or based on conformal theory [13], suggested non classical values. In the recent period however, high resolution simulations 4, 21,6, 19 underlined that, provided long live coherent structures are disrupted, classical behaviour holds ; furthermore, theoretical studies [14,15] suggested the absence of small scale intermittency, placing the direct enstrophy cascade in a position strikingly different from the three-dimensional energy cascade. The recent soap film experiments, developing single point measurements of the velocity field 16,18,17,20], obtained spectral exponents consistent with these views.

Nonetheless, investigating small scale intermittency in this problem requires measuring the statistics of quantities such as the vorticity increments, which has not been done yet, neither in physical nor in numerical experiments. Efforts in this direction were made in the numerical study of Borue [21], but difficulties arose to obtain converged results. An analysis of the enstrophy fluxes in the numerical experiment of Babiano et al [5] led the authors to underlining the presence of weak intermittency in the enstrophy cascade ; thus, although the theory on the problem is at a well advanced stage (at least compared to three-dimensional situation), it is not yet known, even in situations where self similarity fully holds, to what extent classical theory, based on mean field arguments "a la Kolmogorov", can be applied to describe the enstrophy cascade. In the physical experiment we present here, we have extensively measured the statistics of vorticity increments, in a situation where coherent structures have been disrupted. We could show, for the first time, that in the enstrophy cascade, the deviation from gausiannity, for the small scale statistics of the vorticity field, are moderate, and -more importantlyscale independant ; the corresponding structure function exponents are indistinguishable from zero, so that intermittency is absent from the process, in agreement with the theoretical analysis of [15]. This observation, made on a physical system perhaps brings the problem, more firmly, within the reach of a theoretical understanding, a situation rare in the field.

The experimental set-up has been described in a series of papers 25,23,24. It appears that the system we use is a formidable tool for investigating fundamental issues of two-dimensional turbulence. It provides reliable data on quantities reputed hard to measure. We believe this is an interesting situation, since it would be unpleasant to elaborate a rationale for $2 \mathrm{D}$ turbulence, solely on virtual inputs . Briefly speaking, the flow is generated in a square PVC cell, $15 \mathrm{~cm} \times 15 \mathrm{~cm}$. The bottom of the cell is made of a thin (1 mm thick) glass plate, below which permanent magnets, $5 \times 8 \times 4 \mathrm{~mm}$ in size, and delivering a magnetic field, of maximum strength $0.3 \mathrm{~T}$, are placed. In order to ensure two-dimensionality 22], the cell is filled with two layers of $\mathrm{NaCl}$ solutions, $2.5 \mathrm{~mm}$ thick, with different densities, placed in a stable configuration, i.e. the heavier underlying the lighter. Under typical operating conditions, the stratification remains unaltered for pe- 
riods of times extending up to $10 \mathrm{mn}$. The interaction of an electrical current driven across the cell with the magnetic field produces local stirring forces. The flow is visualized by using clusters of $2 \mu \mathrm{m}$ in size latex particles, placed at the free surface, and the velocity fields $\mathbf{v}(\mathbf{x}, t)$ are determined using particle image velocimetry technique, implemented on $64 \times 64$ grids. In such experiments, the dissipative scale for the enstrophy cascade defined by $l_{d}=\eta^{1 / 6} \nu^{1 / 2}$ (where $\nu$ is the kinematic viscosity of the working fluid) - is on the order of $1 \mathrm{~mm}$; it is thus unresolved. Moreover, $l_{d}$ lying below the layer thickness, it is reasonable to consider that the way how enstrophy is dissipated in our system is not purely twodimensional. Concerning now measurement accuracy, we estimate, from the measurement of local divergence, that the accuracy on the velocity is a few percent and that on the vorticity is $10 \%$.

In the experiments we describe here, magnets are arranged into four triangular aggregates of roughly one hundred units, having the same magnetic orientation, as shown schematically in Fig \&. By doing so, the electromagnetic forcing is defined on large scale, and its spatial structure does not favour any particular permanent pattern.

The electrical current is unsteady : it is a non periodic, zero mean, square waveform, of amplitude equal to 0.75 A (see Fig 11). The corresponding Reynolds number defined as the square of the ratio of the forcing to the dissipative scale - is on the order of $10^{3}$; this estimate is one order of magnitude above the largest simulation performed on the subject, using normal viscosity (See [21]). In the statistically steady state, the instantaneous flow pattern consists of transient recirculations of sizes comparable to one fourth of the box size. The formation of permanent large scale structures, which might tend to break the self similarity of the process, seems disrupted by our particular forcing.

The instantaneous vorticity field in the statistically stationary state is shown in Fig 2. We see elongated structures, in form of filaments or ribbons, some of them extending across a large fraction of the cell. At variance with the decaying regimes, and consistently with the above discussion, we have not seen any long live vorticity concentration, i.e persisting more than a few seconds. This is further confirmed by a measurement of the flatness of the vorticity distribution, a diagnostics previously introduced by [6] and which is found slightly above the gaussian value in our case. The presence of coherent structures would have been associated to much larger values of this quantity. The isotropy of the vorticity field is not obvious from the inspection of a single realisation, such as the one of Fig 2 ; nonetheless, as will be shown later, the overall anisotropy level, obtained after statistical averaging, turns out to be reasonably small.

The spectrum of the velocity field, averaged over 200 realisations, in the statistically steady state, is shown in
Fig 3. The forcing wave number $k_{f} \sim 0.6 \mathrm{~cm}^{-1}$ corresponds to the location of the maximum of the energy spectrum ; it is associated to an injection scale $l_{f}=\frac{2 \pi}{k_{f}}$ estimated to $10 \mathrm{~cm}$, a value consistent with the size of our permanent magnets clusters. The wave-number associated to the stratified fluid layer, may be defined as $k_{l}=\frac{2 \pi}{b} \sim 12 \mathrm{~cm}^{-1}$ (where $\mathrm{b}$ is the fluid thickness). This wave-number, together with the sampling wave-number, which is $25 \mathrm{~cm}^{-1}$, are well outside the region of interest. Fig 3 shows that in the high wave number region, i.e above $9 \mathrm{~cm}^{-1}$, the spectrum is flat. This region is dominated by white noise ; it reflects a limitation in the PIV technique to resolve low velocity levels at small scales.

The interesting feature is that there exists a spectral band, lying between $k_{f}$ and $k_{\max } \sim 7 \mathrm{~cm}^{-1}$, uncontaminated by a possible interaction with the layer wavenumber, in which a power law behaviour is observed. The corresponding exponent is close to -3 , as shown on the compensated spectrum. A direct measurement of the exponent, performed by using least square fit in the scaling region, leads to proposing the following formula for the spectrum :

$$
E(k) \sim k^{-3.0 \pm 0.2}
$$

The exponent we find is thus close to classical expectation. There is no steepening effect of the spectrum, which could be attributed, as in decaying systems, to the presence of coherent structures. Further analysis of the vorticity field shows homogeneity and stationary, of the process. Isotropy is also obtained, albeit only roughly, as shown in Fig 1 : to estimate the anisotropy level, we follow circles, embedded in the inertial range, in the spectral plane of Fig 1 , and determine by how much the spectral energy departs from a constant value along such circles. This leads to an anisotropy level on the order of $15 \%$ in the central region of the inertial range ; this is acceptably low. Determining the Kraichnan Batchelor constant is a delicate issue, which entirely relies on the measurement of the enstrophy pumping rate $\eta$. The constant we discuss here, called C', is defined by expressing the energy spectrum in the form :

$$
E(k)=C^{\prime} \eta^{2 / 3} k^{-3}
$$

To measure C', we determine the spectral enstropy transfer rate from below $\mathrm{k}$ to above $\mathrm{k},-\Lambda(k)$ - and search for a plateau, within the scaling range of the energy spectrum. $\Lambda(k)$ is found positive throughout this range, which confirms the cascade is forward. To determine $\eta$, we further average out $\Lambda(k)$, between $k_{f}$ and $k_{\max }$. This procedure provides the following estimate for the Kraichnan Batchelor constant C' :

$$
C^{\prime} \approx 1.4 \pm 0.3
$$

This estimate agrees with that found in the high resolution study of Ref [21], for which values ranging between 1.5 and 1.7 have been proposed. We provide here 
the first experimental measurement ever achieved for this constant.

We now turn to the intermittency problem. Fig 5 shows a set of five distributions of the vorticity increments, obtained for different inertial scales, ranging between 2 and $9 \mathrm{~cm}$. As usual, in order to analyze shapes, the pdfs have been renormalized to impose their variance be equal to unity. The shapes of the pdfs are not exactly the same, but there is no systematic trend with the scale across which the vorticity increment is determined. Within experimental error, the distributions roughly collapse onto a single curve ; the tails of such an average distribution are broader than a gaussian curve, but here again, the deviations have a moderate amplitude and are scale independant. It is thus difficult here, from the inspection of the distributions, to figure out the presence of intermittency in the enstrophy cascade.

The analysis of the structure functions of the vorticity, shown on Fig 6, confirms this statement. These structure functions are defined by :

$$
S_{p}(r)=<(\omega(\mathbf{x}+\mathbf{r})-\omega(\mathbf{x}))^{\mathbf{p}}>
$$

in which $\mathbf{x}$ and $\mathbf{r}$ are vectors, and $\mathrm{r}$ is the modulus of r. The brackets mean double averaging, both in space, throughout the plane domain, and in time, between 20 and $280 \mathrm{~s}$. We use here $10^{5}$ data points to determine the structure functions ; this allows to determine up to twelfth order, because of the near gaussianity of the pdfs. Fig 6 thus represents a series of vorticity structure functions $S_{p}(r)$, obtained in such conditions, emphasizing on the inertial domain, i.e with $\mathrm{r}$ varying between 1 and 10 $\mathrm{cm}$. The structure functions are essentially flat, indicating the structure function exponents are close to zero. The corresponding values of such exponents fall in the range $-0.05,0.15$, for $\mathrm{p}$ varying between 2 and 10 ; this is indistinguishable from zero. We thus obtain here a result fully compatible with the classical theory, for which the exponents are predicted to be exactly zero at all orders. Surprisingly, we do not detect any logarithmic deviation, which would be compatible with the analysis of Refs [15]. It is however not completely safe that this does not reflect a limitation in the measurements.

To summarize, we have performed, for the first time in a physical system, an extensive observation of the enstrophy cascade. Previous experiments inferred its existence from the interpretation of $k^{-3}$ spectra. We provide here a complete observation, along with a measurement of the Kraichnan Batchelor constant, and a determination of the high order vorticity statistics, a crucial quantity to measure for addressing the intermittency problem. We obtain that classical theory is strikingly successful ; there is no sizeable small scale intermittency, and the vorticity statistics departs only weakly from gausiannity, at all scales. Because of these particular features, one may perhaps hope this problem be brought to theoretical understanding. The role of coherent structures, long emphasized on, is indeed important and interesting, but should probably be considered as a separate issue.

This work has been supported by Ecole Normale Supérieure, Universités Paris 6 et Paris 7, Centre National de la Recherche Scientifique, and by EEC Network Contract FMRX-CT98-0175. The authors wish to thank G Falkovitch, V Lebedev, R Benzi for enlightening discussions concerning this study.

[1] R. H. Kraichnan, Phys. Fluids 9, 1937 (1966).

[2] G. Batchelor,Phys Fluids Supp II, 12,233,(1969).

[3] R. Kraichnan, J. Fluid. Mech., 47,3,525(1971).

[4] J.R.Herring, J. Mc Williams J. Fluid. Mech., 153,229,(1985).

[5] A. Babiano, B Dubrulle, $\mathrm{P}$ Frick,Phys Rev E,

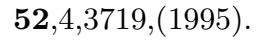

[6] M.E.Maltrud, G.K.Vallis,J. Fluid. Mech., 228,321,(1991).

[7] K. Okhitani,Phys. Fluids, A3,1598,(1991).

[8] M.E.Brachet, M Meneguzzi, P.L. Sulem Phys. Rev. Lett,

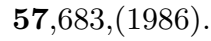

[9] P. Santangelo, B. Legras, R. BenziPhys FLuids A, I,1027 (1989).

[10] B. Legras, P. Santangelo, R. Benzi Europhys. Lett., $\mathbf{5}, 37,(1988)$.

[11] H.K.Moffatt, in Advances in Turbulence, edited by G. Comte-Bellot and J.Mathieu (Springer-Verlag, Berlin, 1986, p 284.

[12] P.G.Saffman, Stud. Appl. Math., 50, 277 (1971).

[13] A. Polyakov, Nucl Phys., B396,367 (1993).

[14] G. Eyink,Phys. D, 91,97,(1996).

[15] G. Falkovitch, V LebedevPhys Rev E, 52,49,3,(1994).

[16] M Gharib, P Derango,Physica D, 37,406,(1989).

[17] B.K.Martin, X.L.Wu, W.I.Goldburg, M.A.RutgersPhys Rev Lett, 80,18,3964,(1995).

[18] Kellay, X-I Wu, W.I. Golburg,Phys Rev Lett,



[19] K.G.Oetzel, G.K.Vallis, Phys Fluids, 9,10,2991,(1997).

[20] M. Rutgers,Phys Rev Lett, 81,11,2244 (1998).

[21] W. Borue, Phys Rev Lett, 71,24,3967 (1993).

[22] J Paret, D. Marteau, O Paireau, P Tabeling, Phys. Fluids, 9, (10), 3102 (1997).

[23] J. Paret and P. Tabeling, Phys. Rev. Lett. 79, 4162 (1997). .

[24] J. Paret and P. Tabeling, Phys. Fluids (1998),10,12,3126.

[25] A. E. Hansen, D. Marteau and P. Tabeling, Phys. Rev.E 58, 7261 (1998). 

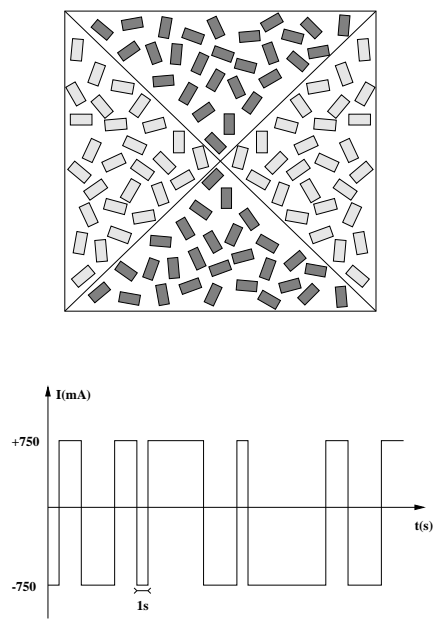

FIG. 1. A sketch of the organisation, in space, of the magnets (as seen from above) and the time dependence of the electrical current crossing the cell. Black units have the same magnetic orientation, grey ones have the opposite one. The averaged laps of time between two successive current switchs is $2.5 \mathrm{~s}$

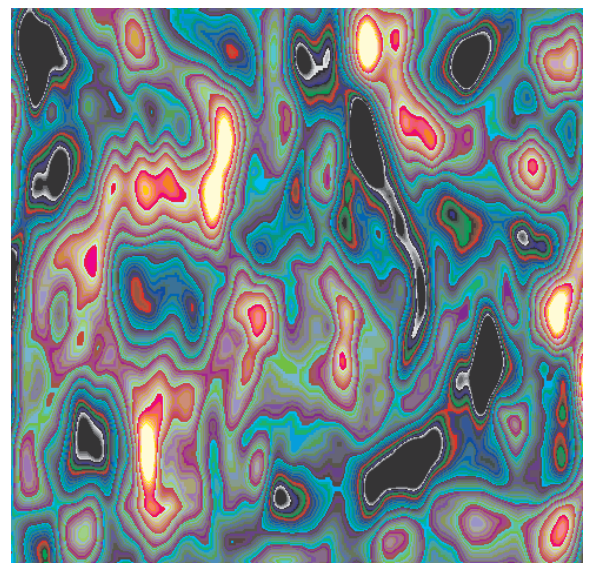

FIG. 2. A particular realisation of the vorticity field, in the statistically steady state ; the grey scale is linear in the vorticity

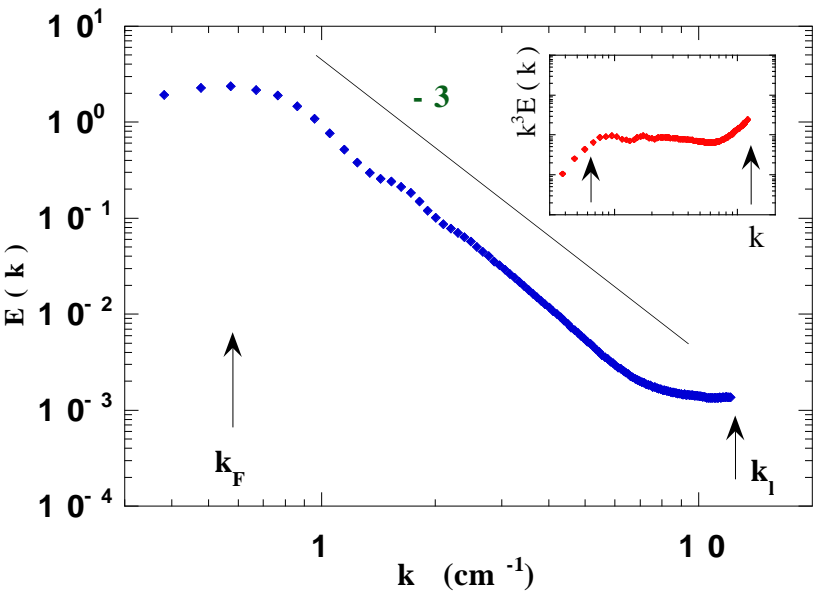

FIG. 3. Energy spectrum of the velocity field, averaged over 200 realisations of the velocity field in the statistically stationary state ; the insert shows the same spectrum, multiplied by $k^{3}$

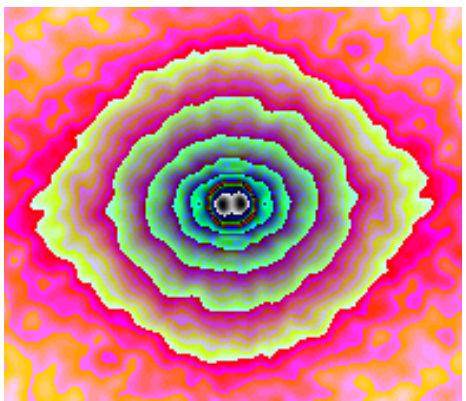

FIG. 4. Two dimensional energy spectrum of the flow under investigation. The two peaks at $k_{x}= \pm 0.6 \mathrm{~cm}^{-1}$ around the center (where $\mathrm{x}$ is the axis crossing these two peaks) signal the forcing. The boundaries of the rectangle, along $\mathrm{x}$ axis corresponds to $k_{x}= \pm 12 \mathrm{~cm}^{-1}$

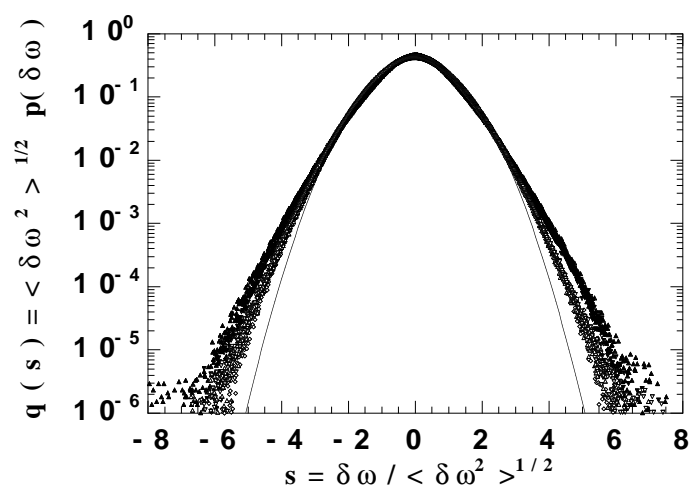

FIG. 5. Normalized distributions of vorticity increments, for five separations $r: 2,3,5,7$ and $9 \mathrm{~cm}$. 


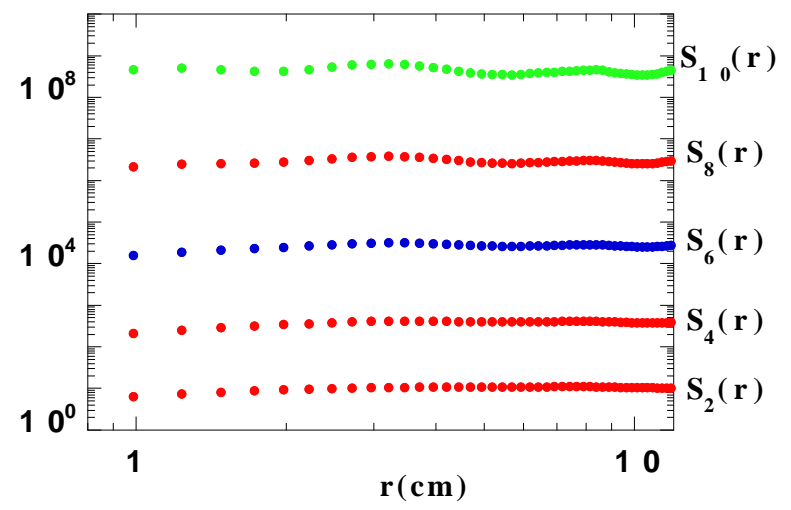

FIG. 6. Structure functions of the vorticity increments, of various orders between 2 and 10 . 